\section{Quadratus lumborum block for postoperative analgesia after abdominal surgery in infant patients: A case series}

Andrus Kõrgvee, $M D^{l}$, Miia Kokkonen, $M D^{l}$, Heikki Koskinen, $M D^{l}$, Eija Junttila, $M D, P h D^{l}$
${ }^{l}$ Tampere University Hospital, Department of Anesthesia, Tampere, Finland

Corresponding author

Andrus Kõrgvee, Department of Anesthesiology, Tampere University Hospital,

PO Box 2000, 33521 Tampere, Finland, tel. +358331169617, e-mail: andrus.korgvee@pshp.fi

\section{Background and aims}

Effective post-laparotomy analgesia is a well-recognised challenge in infant patients. In addition to intravenous opioids various regional analgesic techniques are used, such as epidural blocks with recognised risks and technical difficulties. The recently introduced quadratus lumborum block (QLB) ${ }^{1}$ appears to be an effective analgesic technique after abdominal surgery in adult patients ${ }^{2,3}$. In this case series we report encouraging results using QLB as postoperative analgesia in infant patients who have undergone open abdominal surgery.

\section{Methods}

The data of infant patients who had undergone open abdominal surgery with QLB as regional analgesia was reviewed. In included patients perioperative care followed standard principles: operation was performed under general anesthesia with intravenous fentanyl boluses $1-2 \mu \mathrm{g} / \mathrm{kg}$ for analgesia. Patients received intravenous dexamethasone bolus $0,1-0,25 \mathrm{mg} /$ $\mathrm{kg}$ intraoperatively. Ultrasound-guided QLB (type 2) was performed preoperatively after anesthesia induction, Figure 1 and 2. Postoperatively paracetamol was used regularly and intravenous or peroral opioid (oxycodone or morphine) was available as rescue analgesia. Opioid consumption was reviewed until hospital discharge.

\section{Results}

Five infant patients were included in this series. Patient characteristics, details of QLB performed and opioid consumption are presented in Table 1 and 2. Overall the need for rescue analgesia was minimal. No complications or side effects were detected.

\section{Conclusions}

QLB appears to be an effective regional analgesic technique for postoperative analgesia also in infant patients after open abdominal surgery. Larger prospective studies will be required to establish its efficacy and safety.

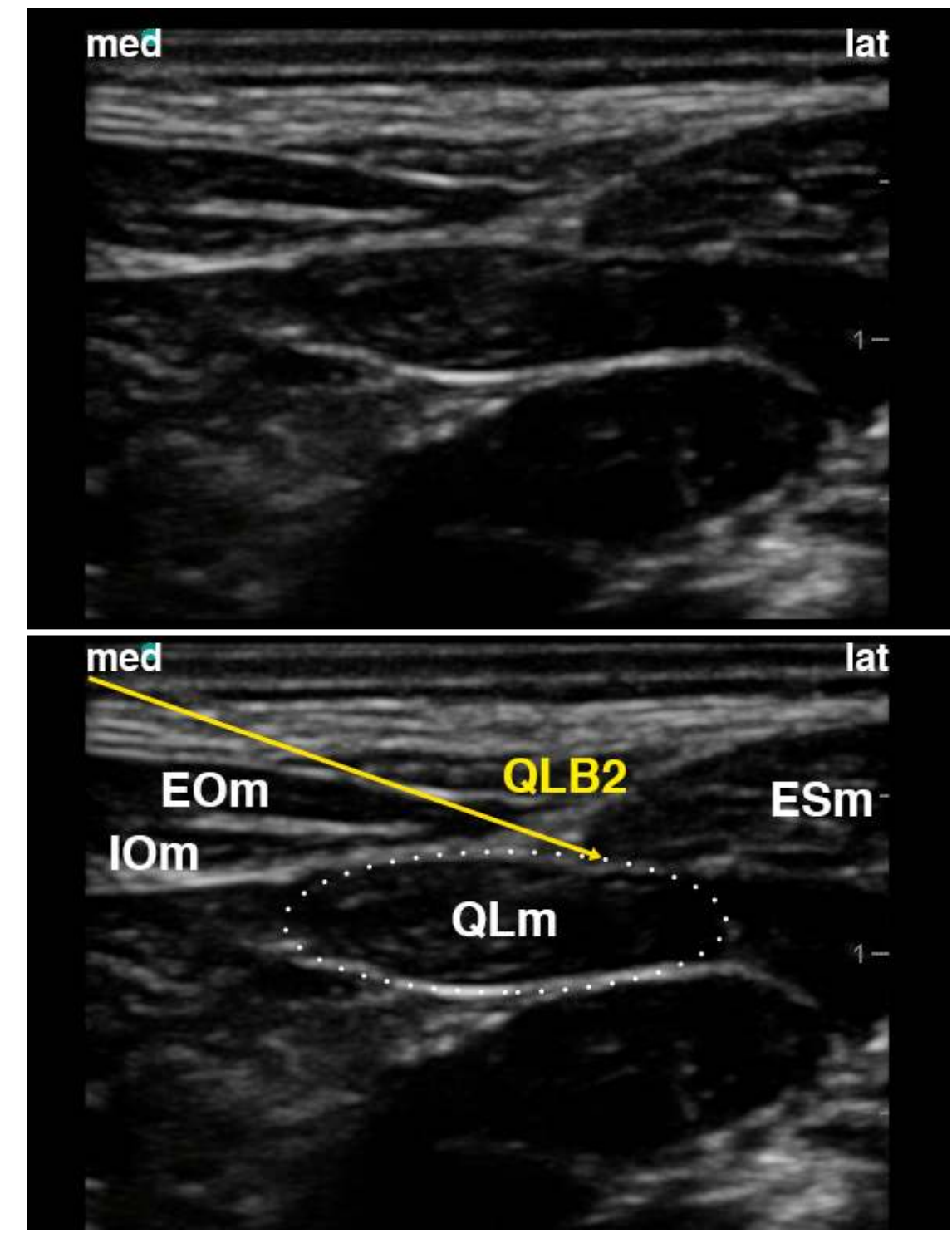

Figure 1. Ultrasound guided QLB2

Abbreviations

EOm external oblique muscle

IOm internal oblique muscle

ESm erector spinae muscle

QLm quadratus lumborum muscle

QLB2 quadratus lumborum block type 2

\begin{tabular}{|c|c|l|c|l|}
\hline Patient & Age, months & Gender & Weight, kg & Operation, incision side and level \\
\hline 1 & 0,6 & male & 4,2 & Laparotomy, left upper quadrant, horisontal \\
\hline 2 & 4 & male & 7,4 & Laparotomy, left upper quadrant, horisontal \\
\hline 3 & 20 & female & 8,0 & Laparotomy, left upper quadrant, horisontal \\
\hline 4 & 1,5 & female & 4,5 & Left, lumbotomy \\
\hline 5 & 1,5 & male & 4,6 & Laparotomy, Pfannenstiel \\
\hline
\end{tabular}

Table 1. Clinical characteristics

\begin{tabular}{|c|l|l|c|c|l|}
\hline Patient & Block side & Local anesthetic & Dose, ml & $\begin{array}{l}\text { Opioid doses } \\
\text { postop, } \mathbf{n}\end{array}$ & Opioid total, mg \\
\hline 1 & Left & Levobupivacaine $1,25 \mathrm{mg} / \mathrm{ml}$ & 3,0 & 2 & Oxycodone 1,6 po \\
\hline 2 & Left & Levobupivacaine $1,25 \mathrm{mg} / \mathrm{ml}$ & 9,0 & 0 & 0 \\
\hline 3 & Left & Ropivacaine $1 \mathrm{mg} / \mathrm{ml}$ & 9,5 & 2 & Oxycodone 0,8 iv \\
\hline 4 & Left & Levobupivacaine $1,25 \mathrm{mg} / \mathrm{ml}$ & 5,0 & 0 & 0 \\
\hline 5 & Left/right & Levobupivacaine $1,25 \mathrm{mg} / \mathrm{ml}$ & 5,2 & 1 & Morphine 0,2 iv \\
\hline
\end{tabular}

Table 2. Block data and opioid consumption

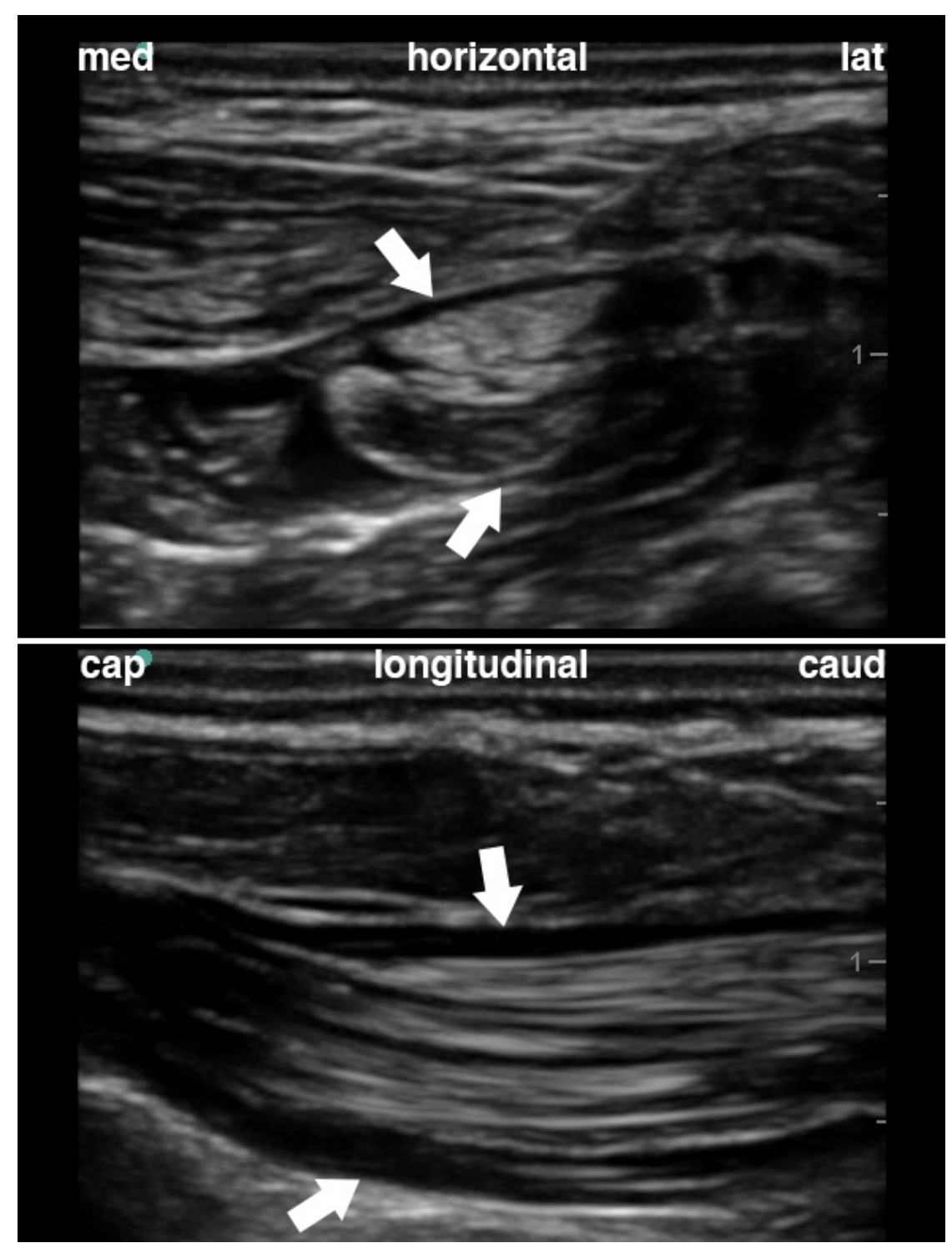

Figure 2. Arrow marks local anesthetic around QL muscle.

\section{$\sum$ Tays}

Tampere University Hospital

\title{
Acknowledgements
}

The expert help of Anna-Riika Hennessy, MD in language issues is much appreciated.

\section{References}

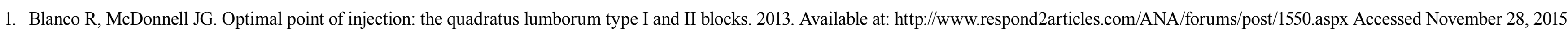


Med: Nov/Dec 2016 - vol 41- $p$ 757-767.

3. Murouchi T, Iwasaki S, Yamakage M. Quadratus lumborum block: analgesic effects and chronological ropivacaine concentrations after laparoscopic surgery. Reg Anesth Pain Med. 2016;41:146-150. 